ARTICLE

\title{
A Fission Matrix Based Methodology for Achieving an Unbiased Solution for Eigenvalue Monte Carlo Simulations
}

\author{
Michael WENNER ${ }^{*}$ and Alireza HAGHIGHAT \\ University of Florida, Gainesville, FL, 32611, USA
}

\begin{abstract}
Recent work in the completely fission matrix based Monte Carlo eigenvalue methodology has posited that the fission matrix elements are independent of the source eigenvector in the limit of small mesh sizes. In this paper, a modified fission matrix based Monte Carlo methodology for achieving unbiased solutions even for high Dominance Ratio (DR) problems is introduced. This new methodology utilizes an initial source, autocorrelation and normality tests, in addition to a Monte Carlo Iterated Confidence Interval (ICI) formulation for estimation of uncertainties in the fundamental eigenvalue and eigenvector. This new methodology is referred to as FMBMC-ICEU (Initial-source Controlled Elements with Uncertainties). The mesh size and particle population were shown to be directly related to the independence of fission matrix coefficients whereby smaller meshing with increasing particle density is necessary to achieve an unbiased solution for the high Dominance Ratio problems. Additionally, it is shown that the ICI algorithm yields a more realistic estimation of uncertainties as compared to the highly conservative plus or minus fission matrix based approach. Lastly the parallel capability of the algorithm was tested and shown to be highly scalable if the number of skipped generations is minimized.
\end{abstract}

KEYWORDS: Monte Carlo, eigenvalue, fission matrix, confidence, bias

\section{Introduction}

Recently, a complete fission matrix based Monte Carlo (FMBMC) methodology was proposed. ${ }^{1-3)}$ In this approach, only the result of a final converged cumulative fission matrix over ALL particles simulated (discarding any skipped generations) is utilized to calculate all desired information. It is shown that the spatial meshing has significant impact on the accuracy of the calculated fission matrix elements, which directly impact the calculated eigenvalue and eigenvector. In the limit of "very small" meshes, the source distribution should be irrelevant in the calculation of the fission matrix elements. As a result, in this extreme case, no skipped generations are necessary and all particles can contribute to the final result.

Practically speaking, however, the mesh size required may make this approach intractable. Moreover, the mesh size necessary to satisfy this "very small" size is unknown. As a result, this paper investigates whether an initialized source distribution with coarser meshing can mitigate some of the mesh size limitation, and applies the fission matrix based methodology to a high Dominance Ratio (DR) problem.

The high DR problems suffer from poor source convergence in which the source distribution appears to have high autocorrelation as a result. This can lead to either biased results or biased confidence/uncertainty estimates of derived parameters. An initial source distribution may provide a good starting source, yet the problem does not converge in the "normal" sense. As a result, in this paper, we develop a

*Corresponding author, E-mail: mtw125@ufl.edu

(C) 2011 Atomic Energy Society of Japan, All Rights Reserved. methodology to assess whether a particular meshing is acceptable.

The new revised Fission Matrix Based Monte Carlo utilizes different techniques for measuring the quality of the elements of the fission matrix, and thereby achieving an unbiased eigenvalue and eigenvector. The new methodology includes four major components: i) An optional algorithm for obtaining a deterministic-based initial source; ii) An autocorrelation algorithm for determination of independence of the FM elements, iii) Formulations for checking the normality of the FM elements, and iv) A Monte Carlo Iterated Confidence Interval (ICI) for determination of uncertainties in the converged eigenvalue and eigenvector. This methodology is referred to as FMBMC with Initial source and Controlled Elements and Uncertainties (FMBMC-ICEU). The FMBMC-ICEU algorithm has been developed for operation on parallel computers, and has been tested on a PC cluster. It is worth noting that diagnostic tests for the fission source convergence are included for testing the source convergence for a 'standard' Monte Carlo eigenvalue approach.

This paper is organized as follows. Section II gives a theoretical discussion on the Fission Matrix Based Monte Carlo (FMBMC) method and modified FMBMC, referred to as FMBMC-ICEU. Section III gives testing and results, and Section IV gives some conclusions and recommendations for future effort.

\section{Theoretical Background}

\section{Fission Matrix Based Monte Carlo (FMBMC)}

The distribution of neutrons in the system $S(\overrightarrow{\boldsymbol{r}})$ is attained from solution of the eigenvalue equation 


$$
S=\frac{1}{k_{\text {eff }}} A S,
$$

where $k_{\text {eff }}$ is the eigenvalue and $A S$ is the next generation source. $A S$ can then be defined as

$$
S(\vec{r})=\frac{1}{k_{\text {eff }}} \int_{V} a\left(\vec{r}^{\prime} \rightarrow \vec{r}\right) d \vec{r}^{\prime} S\left(\vec{r}^{\prime}\right) .
$$

Physically, $a\left(\vec{r}^{\prime} \rightarrow \vec{r}\right)$ is a kernel providing the expected number of fission neutrons produced per unit volume at $\vec{r}$ from fission neutrons at $\vec{r}^{\prime}$. Equation (2) is discretized and the eigenvalue and corresponding eigenvector are obtained by using the power iteration method. ${ }^{4,5)}$ Summing over the total number of mesh cells $(\mathrm{N})$, the discretized form of Eq. (2) is given by

$$
S_{i}=\frac{1}{k} \sum_{j=1}^{N} a_{i, j} S_{j}
$$

where

$$
a_{i, j}=\frac{\int_{V_{i}} d \vec{r} \int_{V_{j}} d \vec{r}^{\prime} a\left(\vec{r}^{\prime} \rightarrow \vec{r}\right) S_{0}\left(\vec{r}^{\prime}\right)}{\int_{V_{j}} d \vec{r}^{\prime} S_{0}\left(\vec{r}^{\prime}\right)} .
$$

The fission matrix elements, $a_{i, j}$ can be thought of as the expected number of fission neutrons born in cell $i$ due to a fission neutron born in cell $j$. Note that the energy dependence has been left out for simplicity. Algorithmically, we have implemented the fission matrix method as follows:

- Discretize the spatial variable (and energy if desired)

- Store the number of source particles in each discretized spatial region as $(\mathbb{C})_{j}$

- At each collision for every particle in the generation, increment a fission neutron counter to add to the proper fission matrix element as:

$(B)_{i, j}=(B)_{i, j}+w \frac{v \Sigma_{f}}{\Sigma_{t}}$.

- After all histories for the current generation have been completed, the fission matrix elements are determined by

$a_{i . j}=\frac{1}{M-n_{s}} \sum_{n=n_{s}+1}^{M} a_{i . j}^{n}$,

where

$a_{i . j}^{n}=\frac{(B)_{i . j}}{(C)_{k}}$,

$M$ is total number of generations and $n_{s}$ is the number skipped generations.

- Calculate the dominant $k_{\text {eff }}$ and corresponding eigenvector by solving

$S_{i}=\frac{1}{k} \sum_{j=1}^{N} a_{i, j} S_{j}$.
- Note that the elements of $a_{i, j}$ correspond to cumulative elements from all active generations

\section{Fission Matrix Based Monte Carlo with Initial Source and Controlled Elements and Uncertainties (FMBMC-ICEU)}

From the previous work on FMBMC, ${ }^{2,3)}$ it is concluded that the FM Method can be effective for solving high DR problems if the fission matrix coefficients (Eq. (3)) are independent of the spatial distribution of the discretized source (Eq. (2)). Further, it was shown that for "small" meshes, the determined FM elements are independent of the fission source. However, the use of "small" meshes may be a daunting task computationally, and requires a methodology for quantification of what is "small".

In this section, a revised methodology is developed and referred to as FMBMC-ICEU, which includes methodologies for obtaining reliable FM elements, and thereby an unbiased eigenvalue and eigenvector.

To obtain reliable fission matrix elements, it is necessary to have a reasonable source distribution, hence in the FMBMC-ICEU, an optional first step was developed in an algorithm for calculation of an initial source distribution using the deterministic PENTRAN ${ }^{6)} \mathrm{Sn}^{7)}$ parallel code for a simplified model. An initialized source is not used in the work for this paper to focus on the potential of the fission matrix element control parameters in solving high DR problems. It is recommended that an initialized source be used in general.

Furthermore, to achieve an accurate set of FM element confidence estimates with simple statistics, it is necessary that each element in one generation is independent of the same element in other generations, which is an indication of statistical randomness; this is a necessity for determining unbiased confidence estimates using the fission matrix method. Moreover, if the source is random, it is expected that a normal distribution would represent the distribution of each element over different generations. Hence, to examine these issues, i.e., quality of FM elements, the FMBMC-ICEU includes an autocorrelation algorithm for testing independence, and algorithms for testing normality. Finally, to obtain a confidence level for the FM elements, we have developed the Monte Carlo Iterated Confidence Interval (ICI). ${ }^{8)}$

In summary, the new FMBMC-ICEU methodology is comprised of the following four components:

(1) Optional Generation of Initial Source

Optionally, an approximate source distribution is obtained via the deterministic Discrete Ordinates PENTRAN code. ${ }^{6}$ This not only provides the ability of obtaining a better set of initial FM elements, but also diminishes the need for skipped cycles/generations.

(2) Test of Independence of FM Elements

In order to identify whether the FM elements are indeed independent, the lag 1 autocorrelation estimate is obtained for each element by

$$
r_{j, k}=\left(\frac{\sum_{m=1}^{M_{a}-1}\left(A_{m}-\bar{A}\right)\left(A_{m+1}-\bar{A}\right)}{\sum_{m=1}^{M_{a}}\left(A_{m}-\bar{A}\right)^{2}}\right)_{j, k} .
$$


- $\quad r_{j, k}$ is the $(j, k)^{\text {th }}$ fission matrix element lag 1 autocorrelation estimate

- $M_{a}$ is the total number of active generations

- $A_{i}$ is the fission matrix element for $i$ 'th generation

The calculated lag 1 autocorrelation coefficient is compared with the expected lag 1 correlation coefficient for a standard normal distribution with mean equal to the average coefficient and standard deviation equal to the sample standard deviation.

\section{(3) Normality Test}

If the FM elements are obtained from a truly random source distribution, then it is necessary that the elements follow a normal distribution for all active fission generations. Hence, the FMBMC-ICEU includes the use of normality tests, including the Shapiro-Wilk, Cramer-Von-Mises and Kolmgorov-Smirnov tests. ${ }^{9)}$ Note that for very large sample sizes $(>1,000)$, the power of the normality tests may increase to diagnose even insignificant departures from normality. If the FM elements are indeed independent, then the large sample size should ensure that the average element follows a normal distribution at least approximately.

(4) Uncertainty/Confidence of Eigenvalue and Eigenvector

In order to determine the confidence level on the calculated eigenvalue and eigenvector, we have applied the Monte Carlo Iterated Confidence Interval (ICI) technique, which is comprised of the following steps:

1. After converging on the FM elements, and calculating the corresponding $\boldsymbol{k} \& \boldsymbol{S}$, we estimate the uncertainty range for each FM element, considering the Central Limit Theorem (CLT) by

$\left(\varepsilon_{i, j}^{2}\right)_{\underline{\underline{A}}}=\frac{\left(\varepsilon_{i, j}^{2}\right)_{\underline{A}}}{N}$,

where

$\left(\varepsilon_{i, j}^{2}\right)_{\underline{\underline{A}}}=\frac{1}{M-n_{s}-1} \sum_{m=n_{s}+1}^{M}\left(a_{i j, m}-\bar{a}_{i, j}\right)^{2}$.

2. Repeat the following steps for a large number of times $(n)$ :

a. Using a normal distribution with $\bar{a}_{i, j}=$ mean and $\left(\varepsilon_{\mathrm{i}, \mathrm{j}}^{2}\right)_{\underline{\mathrm{A}}}=\sigma$, sample new $a_{i j}$ and obtain a new fission matrix.

b. Calculate a new eigenvalue and corresponding eigenvector.

3. The Percentile Method ${ }^{10)}$ is used to form a confidence level (CL) and estimate the uncertainty in the calculated eigenvalues and each element of the eigenfuctions; i.e., the interval $\delta k=k_{2}-k_{1}$, where $k_{1}=(n+1) \alpha, k_{2}=(n+1)(1-\alpha)$ and $C L=1-2 \alpha$. Note that $i_{f}$ the distribution is skewed, a correction procedure should be applied.

\section{Testing and Results}

\section{Algorithm Implementation}

The fission matrix algorithm was implemented in a 1-Dimensional multigroup Monte Carlo code written in
FORTRAN90/95 with both a traditional solution algorithm and FMBMC-ICEU. The solution algorithms compute $k_{\text {eff }}$ utilizing a standard collision estimator. In the current algorithm, for simplicity, the scattering interaction is considered isotropic. Optionally, an initialized source is determined by the PENTRAN ${ }^{6}$ 3-D, parallel $\mathrm{S}_{\mathrm{N}}$ code through the use of a separate utility code.

Although it is easy to calculate the resulting $k_{\text {eff }}$ and source eigenvector from the final converged fission matrix, determination of the final confidence/uncertainty of the eigenvalue and corresponding source eigenvector is not as straightforward. Dufek ${ }^{2)}$ proposed the use of the plus and minus fission matrix as bounding values, where the plus fission matrix correspond to adding the variance estimate to each fission matrix element for the entire fission matrix, and the same but subtracting for the minus fission matrix given by

$$
\begin{aligned}
& (\mathbb{A})_{i, j}^{+}=(\mathbb{A})_{i, j}+s_{\bar{A}_{i, j}}, \\
& (\mathbb{A})_{i, j}^{-}=(\mathbb{A})_{i, j}-s_{\bar{A}_{i, j}} \forall i, j .
\end{aligned}
$$

The resultant eigenvalues and eigenvectors for the plus and minus fission matrix then form a very conservative confidence bound. This confidence interval will be referred to as the "conservative FMBMC interval in our 1-D algorithm, we have implemented both Eq. (8) and the ICI methodology discussed in Section II.

\section{Test Problem and Results}

\section{(1) Problem Description}

The first test problem is a simple monoenergetic single slab problem with vacuum boundaries and is in a slightly supercritical configuration. It was chosen since it has been studied before and it was determined to have a dominance ratio of $\sim 0.991$. $^{11)}$ Geometry and material properties are shown in Fig. 1.

The slab is in a slightly supercritical state. Analysis in reference 12 indicates that $k_{\text {eff }}$ is approximately 1.02082 .

Importance related to neutron production was calculated with PENTRAN for test problem 1. The resulting importance to production is shown in Fig. 2.

From Fig. 2, it is apparent that the importance to production for this problem is very flat, except near the problem boundary. Source coupling within the slab should not be a problem. An initialized source was not utilized here to benchmark the other features of the FMBMC-ICEU

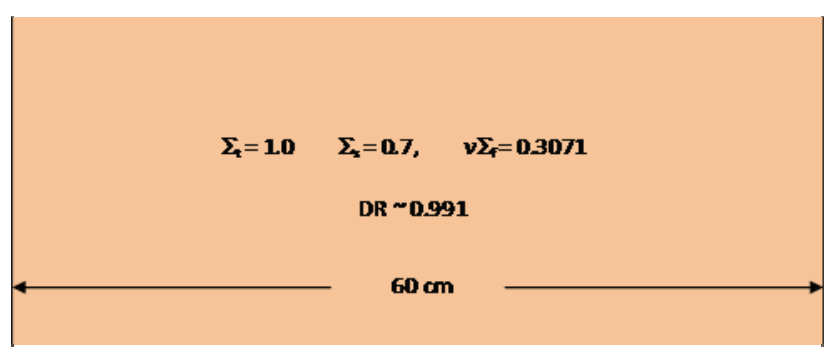

Fig. 1 Geometry and Material Data for Test Problem 1 


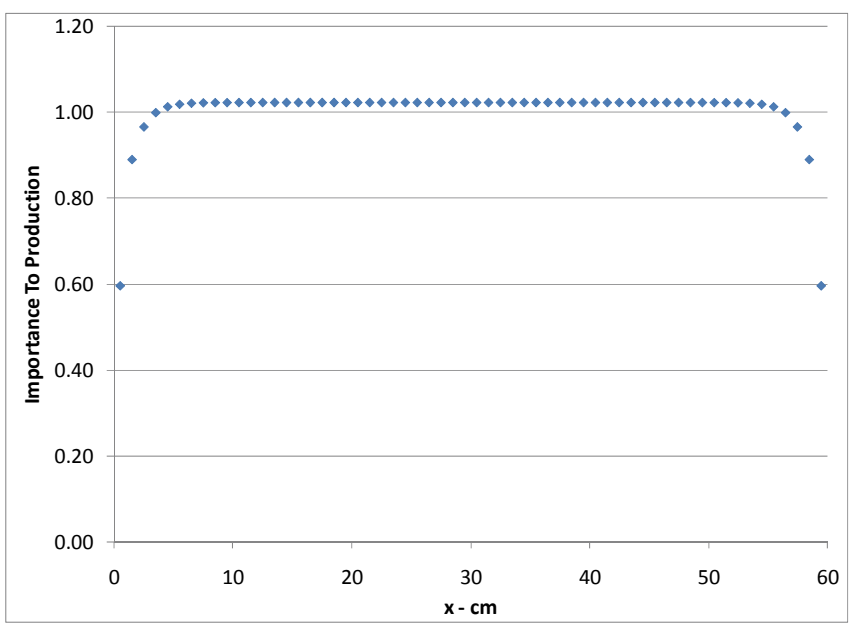

Fig. 2 Importance to Production for the Test Problem

Table 1 Replication Input Parameter Summary

\begin{tabular}{lllll}
\hline Case & $\begin{array}{l}\text { Source } \\
\text { Meshes }\end{array}$ & $\begin{array}{l}\text { Skipped } \\
\text { Gens. }\end{array}$ & $\begin{array}{l}\text { Neutrons } \\
\text { per Gen. }\end{array}$ & $\begin{array}{l}\text { Total } \\
\text { Gens. }\end{array}$ \\
\hline $1-10$ & 5 & 350 & $50 \mathrm{k}$ & 5350 \\
$11-20$ & 12 & 350 & $50 \mathrm{k}$ & 5350 \\
$21-30$ & 12 & 350 & $100 \mathrm{k}$ & 5350 \\
$31-40$ & 24 & 350 & $100 \mathrm{k}$ & 5350 \\
$41-50$ & 24 & 350 & $100 \mathrm{k}$ & 20350 \\
\hline
\end{tabular}

algorithm, therefore 350 generations were skipped in all test cases to ensure (assumption) source settling. Table 1 contains a list of all cases (groups of replications) simulated.

Although the purpose of this methodology is to provide accurate localized data, $k_{\text {eff }}$ is shown for comparison for all cases in Fig. 3.

Figure 3 shows that the $k_{\text {eff }}$ values are fairly consistent. At 1 sigma, both the standard method and the FMBMC-ICEU method yield similar results, yet the error bars for the FMBMC-ICEU method are slightly wider. This could be in part due to the effect of the high dominance ratio. Also note the difference between the reported $k_{\text {eff }}$ value in Reference 11

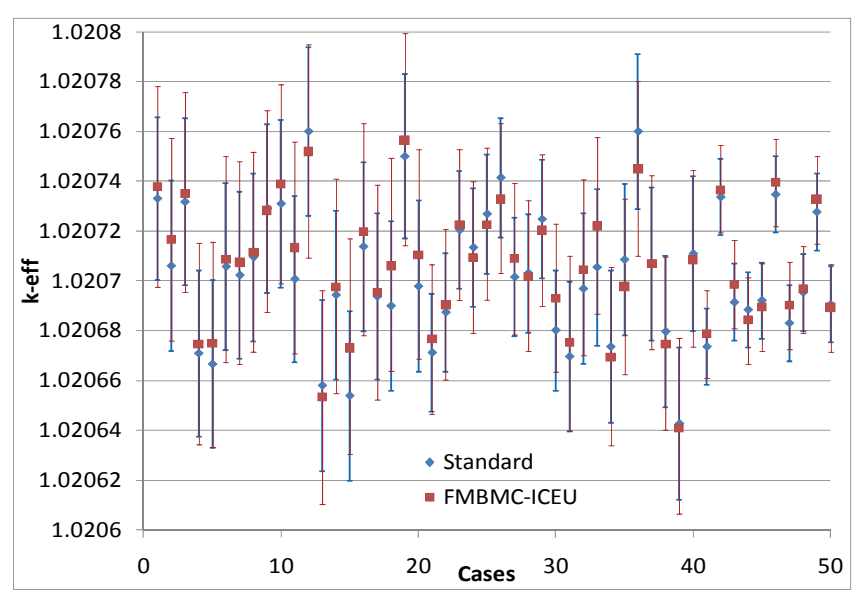

Fig. 3 The $k_{\text {eff }}$ values for different cases

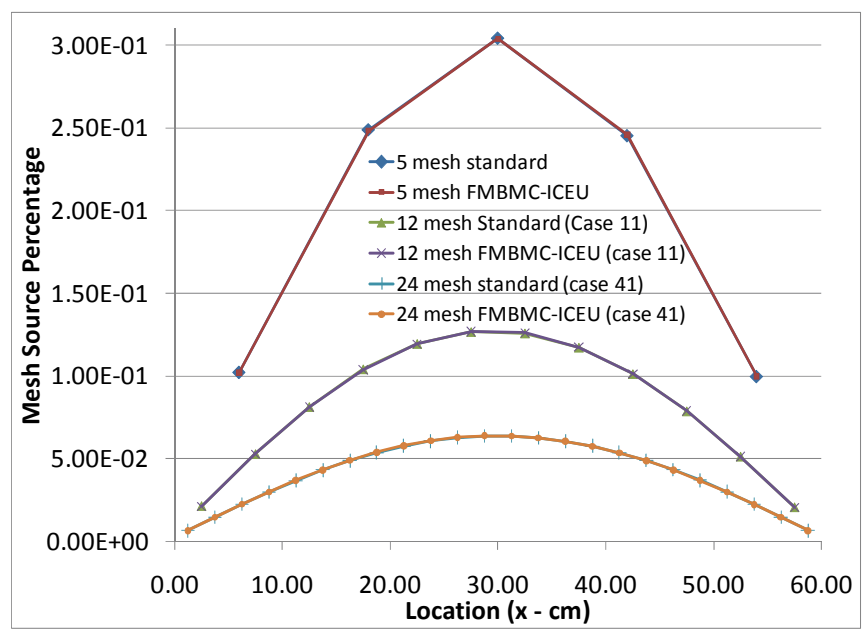

Fig. 4 Representative Converged Source Bins for Different Input Cases

(1.02082). Independent PENTRAN results for this problem yielded a $k_{\text {eff }}$ of 1.020691 , more consistent with the results shown in this work. To continue the analysis where the source is concerned, the source distributions for replications 1, 11, and 41 are shown in Fig. 4.

Figure 4 shows that visually, the results appear symmetric and therefore some measure of accuracy is expected. Other replications yield similar results.

Contrasting this result, however is the fact that the independent $\mathrm{KPSS}^{12)}$ source convergence diagnostic indicates possible source convergence difficulty in 44 of the 50 replications. To see this fact, the source Center of Mass (COM) is shown in Fig. 5 for a representative replication. Figure 5 shows typical source behavior for a high DR problem. The source is highly correlated for which source convergence is difficult to identify, and confidence estimation more complex.

In order to see the impact of the source behavior on the tallied results, source mesh 1 is plotted for both the standard and FMBMC-R method for all cases. Cases 1-10 are shown

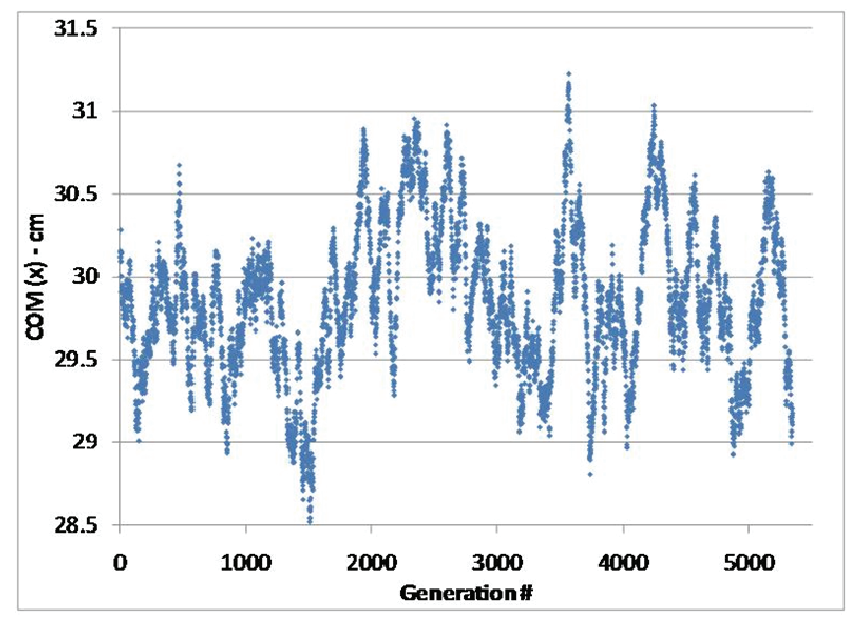

Fig. 5 Source Center of Mass for one Replication, Representative of All Replications 


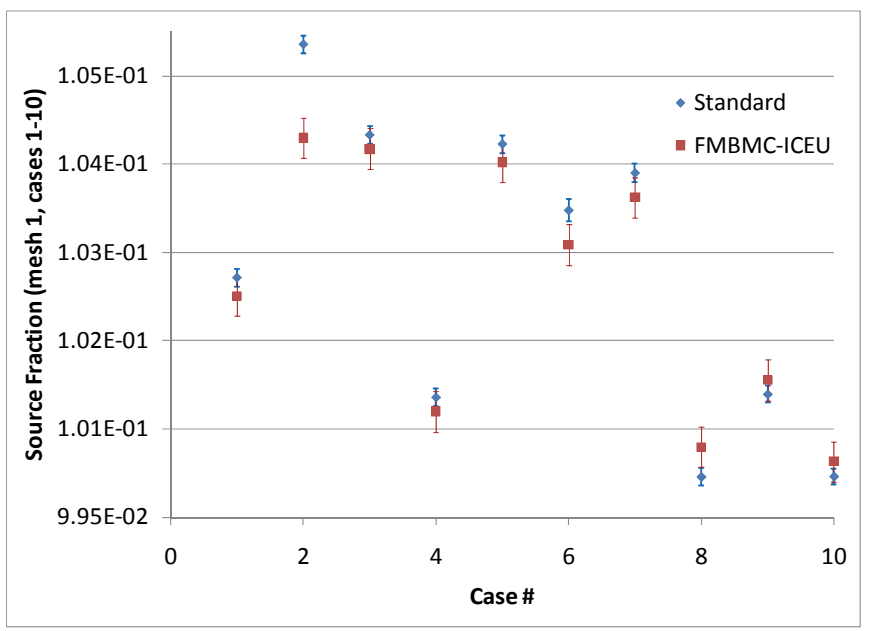

Fig. 6 Mesh 1 Source Fraction for Cases 1-10 for the standard and FMBMC-ICEU methodologies

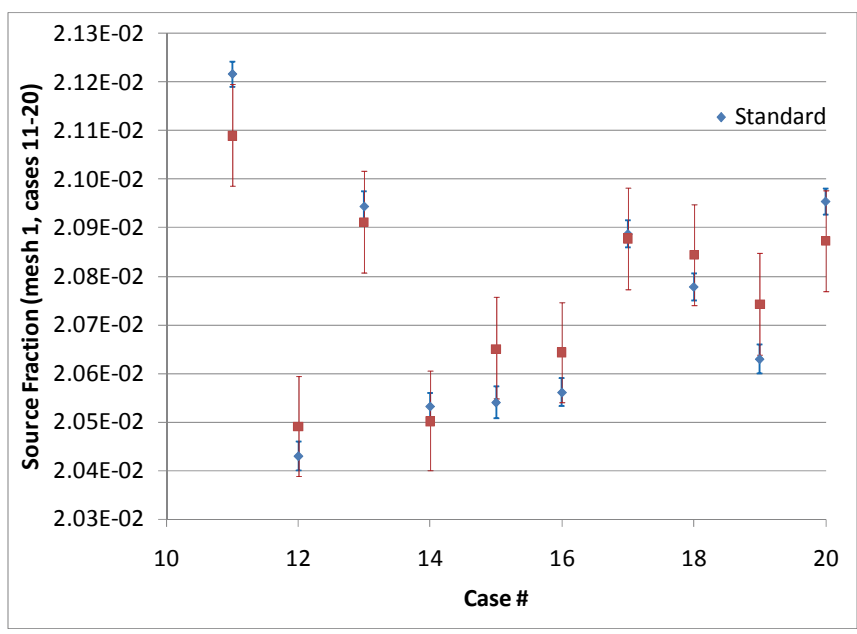

Fig. 7 Mesh 1 Source Fraction for Cases 11-20

in Fig. 6. Note that all figures with error bars show 1- $\sigma$ confidence.

Cases 1-10 represent the coarsest source meshing with $12 \mathrm{~cm}$ source meshes. Figure 6 shows that the final obtained source fraction from mesh 1 for the ten replications are inconsitent even for $2 \sigma$. While the overall variation in the data is small, the calculated uncertainty for both the standard and FMBMB-ICEU method are erroneous. Cases 11-20 are shown in Fig. 7.

Cases 11-20 represent an increase of 5 to 12 source meshes compared to cases 1-10. Figure 7 shows that the final obtained source fraction from mesh 1 for the ten replications are again inconsitent for several cases even at $2 \sigma$. Cases 21-30 are shown in Fig. 8.

Cases 21-30 represent an increase of particle per generation compared to cases 11-20. Figure 8 shows that the final obtained source fraction from mesh 1 for the ten replications are again inconsitent for the standard method, yet consistency has improved for the FMBMC-ICEU results, yet results still appear inconstent at $1 \sigma$. Cases 31-40 are shown in Fig. 9.

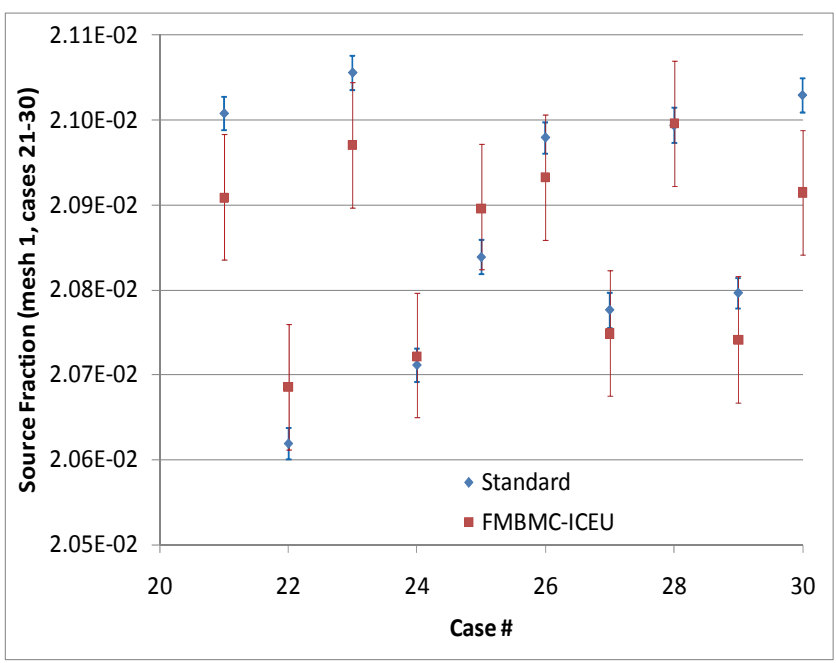

Fig. 8 Mesh 1 Source Fraction for Cases 21-30

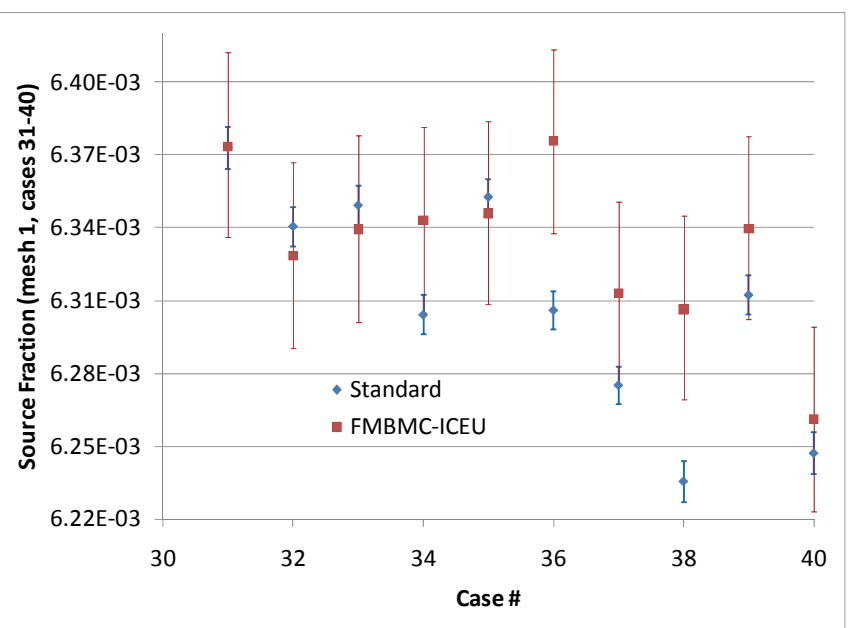

Fig. 9 Mesh 1 Source Fraction for Cases 31-40

Cases 31-40 represent an increase in the number of fission matrix meshes to 24. Figure 9 shows that the final obtained source fraction from mesh 1 for the ten replications are again inconsistent for the standard method, yet the FMBMC-ICEU results appear acceptable. To reduce the size of the error bars for the FMBMC-ICEU results further, Cases 41-50 are shown in Fig. 10 for an increased total active generations from 5,000 to 20,000.

Figure 10 shows that the final obtained source fraction from mesh 1 for the ten replications is still inconsistent for the standard method.

Since independence of the fission matrix coefficients is required in order to accurately calculate the confidence estimates for the eigenvalue and eigenvector, the autocorrelation coefficient of the $A_{12}$ element is shown for cases 1, 11, 21, 31 and 41 in Fig. 11.

Figure 11 illustrates the effect of source discretization and density on the $A_{12}$ fission matrix element. Elements 1 off of the diagonal often have the highest correlation, which is why this element was chosen for illustration. As mesh size is decreased, the correlation is reduced. Note that for cases 11 and 21 , the same size fission matrix is utilized with different 


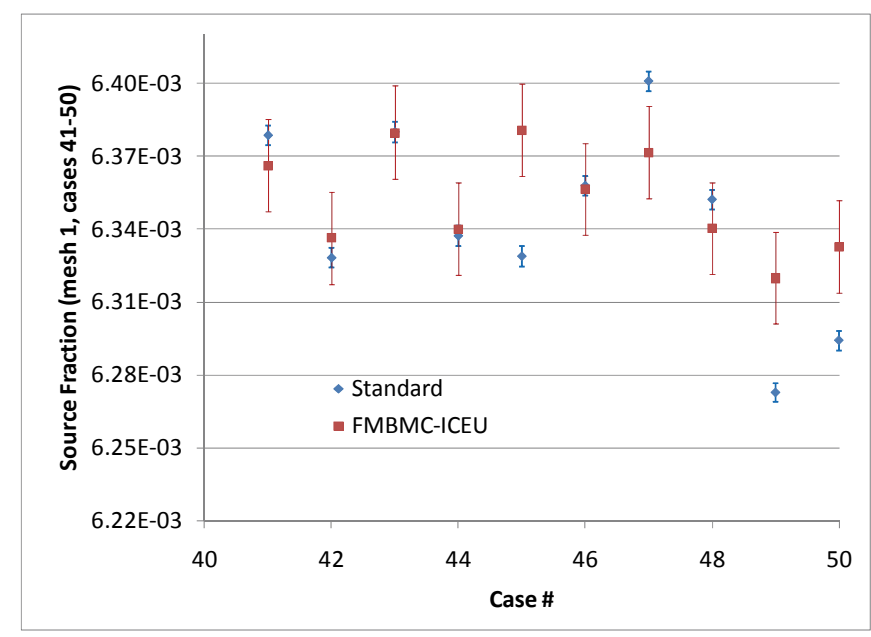

Fig. 10 Mesh 1 Source Fraction for Cases 41-50

particles per generation, yet the fission matrix autocorrelation is not changed significantly. Cases 31 and 41 indicate that increasing the number of generations has little effect on the autocorrelation.

Recall that in order to form accurate confidence intervals, not only do the coefficients need to be uncorrelated, but they must follow a normal distribution. With such large sample sizes, however, normality tests are often unreliable as they identify insignificant deviations from the Normal distribution. This is because with a large sample size, normality tests with have enough data at hand to identify very small deviations from normality (statistically overpowered test). Even if the coefficient being sampled is drawn from a normal distribution, the sample itself may at times appear slightly non-normal.

Fortunately, for large sample sizes, so long as the fission matrix correlation is small, the CLT should apply and the fission matrix elements average values should tend toward a normal distribution and be accurately quantified. A Shapiro-Wilk Normality test was utilized, however, for each coefficient, but is even more informative for smaller sample sizes.

Using the final converged fission matrix, it is possible to estimate the fraction of the final $k_{\text {eff }}$ obtained from non-independent fission matrix coefficients (at a chosen significance). If the lag 1 autocorrelation is too high (as compared to a standard normal curve with the same mean and standard deviation), the element is considered correlated and its' contribution to the final $k_{\text {eff }}$ is estimated by $A_{i, j} * S$ (S fractional source). A similar contribution from non-normal fission matrix elements can be calculated (at a chosen significance) utilizing the Shapiro-Wilk (or another) normality test.

For case 1, it was determined that all of the fission matrix elements but one were not independent. This corresponded to an approximate $100 \%$ contribution from correlated elements. The $k_{\text {eff }}$ for non-normal meshes was determined to be $\sim 13 \%$. Note that if the fission matrix coefficient series is correlated, the normality test for that mesh is not valid. Comparatively, for case 41, the approximate

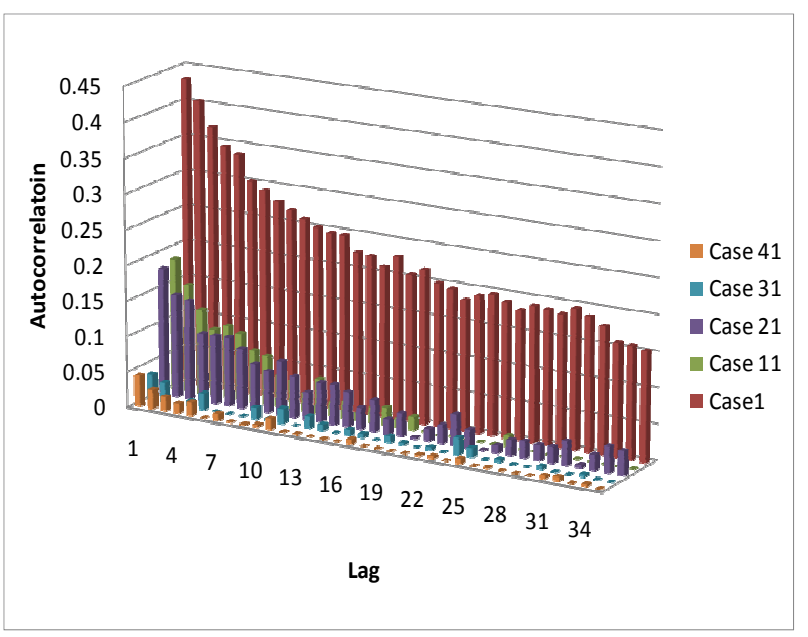

Fig. 11 Autocorrelation of the $A_{12}$ Fission Matrix Coefficient for Representative Cases

non-independent percentage was reduced to $17 \%$, while the non-normal percentage was $26 \%$.

\section{Parallel Algorithm and Speedup}

To show the scalability and inherent parallel nature of the FMBMC-ICEU approach, a parallel algorithm was developed without the use of MPI or PVM. Speedup can be obtained easily as a ratio of figure of merits:

$$
\text { speedup }=\frac{F O M_{2}}{F O M_{1}}
$$

where

$$
F O M=\frac{1}{R^{2} T}
$$

and $R$ is relative error and $T$ is simulation time.

A driver code was written to start jobs through $\mathrm{SSH}$ on LINUX and gather the results of all the fission matrix results into one fission matrix. In order to calculate the combined $k_{\text {eff }}$ and associated confidence interval, each fission matrix coefficient standard deviation is combined and the final fission matrix sampled. Simply combining the individual $k_{\text {eff }}$ values may result in a biased result since the distribution of the resulting eigenvalue and eigenvector from the Iterated-MC confidence interval does not necessarily follow a normal distribution.

For this demonstration a simple infinite slab problem was simulated with 3 and 5 processors, keeping active generations constant. The speedups obtained using Eq. (13) for 3 and 5 processors were 2.57 and 3.71 , respectively. Since 350 skipped generations were utilized, each processor is also performing 350 skipped generations, and a penalty in parallel speedup is taken due to this. If an initialized source is being utilized (which for this problem is the case), skipped generations are unnecessary (or minimally necessary). The estimated extra time spent on skipped generations for each parallel case was calculated and removed from the total time to approximate the ideal speedup case without the loss of speedup from skipped generations. The adjusted ideal speedups for the two cases increased to 2.91 and 4.68. The 


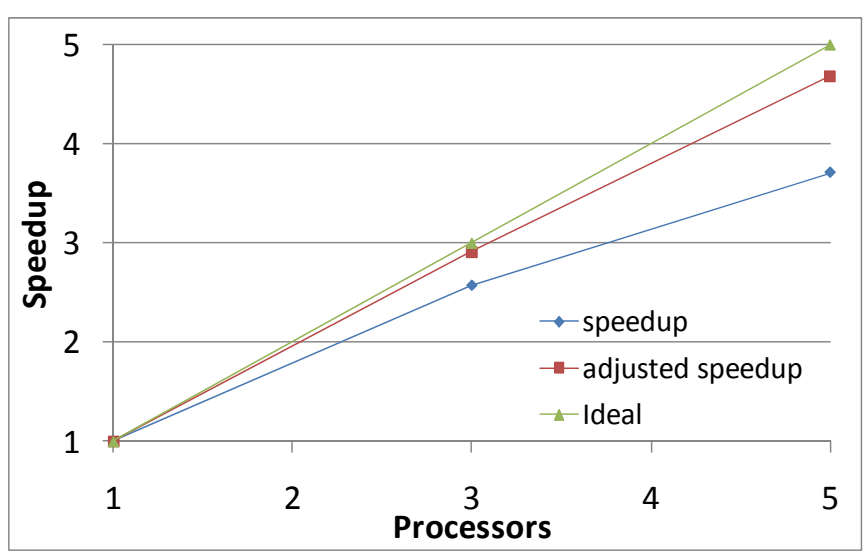

Fig. 12 Speedup Comparison for 1, 3 and 5 processors

results of parallel performance of the FMBMC-ICE are shown in Fig. 12.

Figure 12 shows that speedup is near linear when corrected for the effect of skipped generations, while when skipped generations are a significant portion of the simulation, speedup suffers as can be seen for the lower than ideal speedup obtained for the 5 processor case.

\section{Conclusion and Future Effort}

A new fission matrix based Monte Carlo approach (referred to FMBMC-ICEU) has been developed and utilized to calculate the eigenvalue $\left(k_{e f f}\right)$ and eigenvector (source distribution) for a high DR problem.

This approach utilized a new confidence estimation approach called Monte Carlo Iterated Confidence Intervals. The results are a much improved confidence estimate instead of the much more conservative plus and minus fission matrix as has been previously applied with the fission matrix based approach.

Results concluded that increasing fission matrix meshes resulted in a severe reduction in correlation in the fission matrix elements. It was shown that the source obtained with increasing fission matrix coefficients is consistent among independent replications as long as enough meshes are used to mitigate the correlation. Lastly, the FMBMC-ICEU method was tested in parallel, and it was demonstrated that the algorithm indicates the potential for high scalability when the number of skipped generations is minimal, which will be the case when using an initialized source.

Future effort should focus on the seemingly important link between the particle density and the fission matrix mesh density.

Since a large number of histories are needed, it is necessary to accelerate the sampling of FM elements; We have already developed methods based on the CADIS methodology (i.e., the use of importance function for source and transport biasing). Further studies are needed to learn of limitations of the FMBMC-ICEU.

\section{References}

1) J. Dufek, "Accelerated Monte Carlo eigenvalue calculations," Proc. of Reactor Physics Calculations in the Nordic Countries, (2007).

2) J. Dufek, Development of new Monte Carlo methods in reactor physics, criticality, non-linear steady-state and burnup problems, Ph.D. dissertation, Royal Institute of Technology, Stockholm, Sweden, 2009.

3) J. Dufek, W. Gudowski, "Fission matrix based Monte Carlo criticality calculations," Ann. Nucl. Energy, 36[8], 1270-1275 (2009).

4) E. E. Lewis, W. F. Miller, Computational Method of Neutron Transport,: American Nuclear Society, La Grange Park, IL (1993).

5) G. I. Bell, S. Glasstone, Nuclear Reactor Theory, Robert E. Krieger Publishing, Malabar, FL (1985).

6) B. G. Carlson, Solution of the transport equation by the $S N$ approximations, LA-1599 Los Alamos National Laboratory (LANL) (1953).

7) G. Sjoden, A. Haghighat, "Computer Code Abstract: PENTRAN - Parallel Environment Neutral-particle TRANsport in 3-D Cartesian Geometry," Proc. of the Joint International Conference on Mathematics and Supercomputing for Nuclear Applications, Saratoga Springs, New York, 1, .232-234S (1997).

8) T. Buckland, "Monte Carlo confidence intervals," Biometrics, 40, 811-817, (1984).

9) H. C. Jr. Thode, Testing for Normality (Statistics: a Series of Textbooks and Monographs), CRC Press (2002).

10) B. Efron, "The jackknife, the bootstrap, and other resampling plans," Society of Industrial and Applied Mathematics CBMS-NSF Monographs, 38 (1982).

11) T. J. Urbatsch, Iterative Acceleration Methods for Monte Carlo and Deterministic Criticality Calculations, Ph.D. dissertation, Los Alamos National Laboratory (Univ. of Michigan) (1995)

12) M. Wenner, A. Haghighat, "A generalized KPSS test for stationarity detection in Monte Carlo eigenvalue problems," Proc. PHYSOR 2008 (2008). 